\title{
Investigations on Average Fluorescence Lifetimes for Visualizing Multi-Exponential Decays
}

\author{
Yahui $L^{1,2,3}{ }^{3}$, Sapermsap Natakorn ${ }^{4}$, Yu Chen ${ }^{4}$, Mohammed Safar ${ }^{1}$, \\ Margaret Cunningham ${ }^{1}$, Jinshou Tian ${ }^{2,3}$ and David Day-Uei Li ${ }^{1 *}$
}

${ }^{1}$ Strathclyde Institute of Pharmacy and Biomedical Sciences, University of Strathclyde, Glasgow, United Kingdom, ${ }^{2}$ Key Laboratory of Ultra-fast Photoelectric Diagnostics Technology, Xi'an Institute of Optics and Precision Mechanics, Xi'an, China, ${ }^{3}$ University of Chinese Academy of Sciences, Beijing, China, ${ }^{4}$ Department of Physics, Scottish Universities Physics Alliance, University of Strathclyde, Glasgow, United Kingdom

OPEN ACCESS

Edited by:

Klaus Suhling,

King's College London,

United Kingdom

Reviewed by:

Nirmal Mazumder,

Manipal Academy of Higher

Education, India

Ilia L. Rasskazov,

University of Rochester, United States

*Correspondence:

David Day-Uei L

david.li@strath.ac.uk

Specialty section:

This article was submitted to

Optics and Photonics,

a section of the journal

Frontiers in Physics

Received: 27 July 2020 Accepted: 09 September 2020

Published: 16 October 2020

Citation:

Li Y, Natakorn S, Chen Y, Safar M, Cunningham M, Tian J and Li DD-U

(2020) Investigations on Average

Fluorescence Lifetimes for Visualizing

Multi-Exponential Decays.

Front. Phys. 8:576862.

doi: 10.3389/fphy.2020.576862
Intensity- and amplitude-weighted average lifetimes, denoted as $\tau_{l}$ and $\tau_{A}$ hereafter, are useful indicators for revealing Förster resonance energy transfer (FRET) or fluorescence quenching behaviors. In this work, we discussed the differences between $\tau_{l}$ and $\tau_{A}$ and presented several model-free lifetime determination algorithms (LDA), including the center-of-mass, phasor, and integral equation methods for fast $\tau_{l}$ and $\tau_{A}$ estimations. For model-based LDAs, we discussed the model-mismatch problems, and the results suggest that a bi-exponential model can well approximate a signal following a multi-exponential model. Depending on the application requirements, suggestions about the LDAs to be used are given. The instrument responses of the imaging systems were included in the analysis. We explained why only using the $\tau_{\text {l }}$ model for FRET analysis can be misleading; both $\tau_{l}$ and $\tau_{A}$ models should be considered. We also proposed using $\tau_{A} / \tau_{l}$ as a new indicator on two-photon fluorescence lifetime images, and the results show that $\tau_{A} / \tau_{l}$ is an intuitive tool for visualizing multi-exponential decays.

Keywords: fluorescence lifetime imaging, lifetime determination algorithm, average lifetimes, multi-exponential decays, lifetime image visualization, FRET-fluorescence resonance energy transfer

\section{INTRODUCTION}

Fluorescence lifetime imaging (FLIM) is a crucial technique for assessing microenvironments of fluorescent molecules [1, 2], such as $\mathrm{pH}[3], \mathrm{Ca}^{2+}[4,5], \mathrm{O}_{2}$ [6], viscosity [7], or temperature [8]. Combining with Förster Resonance Energy Transfer (FRET) techniques, FLIM can be a powerful "quantum ruler" to measure protein conformations and interactions [9-12]. Compared with fluorescence intensity imaging, FLIM is independent of the signal intensity and fluorophore concentrations, making FLIM a powerful quantitative imaging technique for applications in life sciences [13], medical diagnosis [14-16], drug developments [17-19], and flow diagnosis [20-22]. FLIM techniques can build on time-correlated single-photon counting (TCSPC) [23-25], time-gating [26-28], or streak cameras [29]; they record time-resolved fluorescence intensity profiles to extract lifetimes with a lifetime determination algorithm (LDA) [1]. There is a rapid growth of real-time applications that fast analysis is sought after $[12,30]$. Traditional LDAs usually use the least square method (LSM) or maximum likelihood estimation (MLE) [31] to analyze decay models chosen by users, and model-fitting analysis follows a reduced chi-squared criterion [1]. In reality, however, it is difficult to know the exact decay model as fluorescent molecules in biological systems can demonstrate 
complex multi-exponential decay profiles. For instance, a mixture of fluorophores, a multi-tryptophan protein, single fluorophores in varied environments, and single-tryptophan proteins in multiple conformational states [1] can show multiexponential decays as

$$
f(t)=A \sum_{i=1}^{p} q_{i} \exp \left(-t / \tau_{i}\right), \text { where } \sum_{i=1}^{p} q_{i}=1
$$

where $A$ represents the amplitude, $q_{i}$ and $\tau_{i}(i=1, \ldots$, $p$ ) denote the amplitude fractions and lifetimes, respectively, and $p$ is the number of lifetime components. There are time-domain or frequency-domain [32-35] FLIM systems to measure a fluorescence decay. In this work, we focus on timedomain approaches.

Suppose the instrument response function (IRF) of the measurement system is $\operatorname{irf}(t)$, the task performed by FLIM analysis tools is to extract $f(t)$ from the measured decay $h(t)$, as

$$
h(t)=\operatorname{irf}(t) * f(t)
$$

The problems with traditional LSM or MLE are two-fold. (1) It is challenging to categorize a fluorescence emission into a specific exponential model described by Equation (1) in complex biological processes. An arbitrary choice of $p$ in Equation (1) simply based on reduced chi-squared tests [36] would lead to totally different interpretations. As the fitting routine is not mathematically unique; a measured decay could be fitted equally well with a bi-exponential or a tri-exponential model. (2) To ensure the accuracy, it usually needs a high photon count (long acquisition time) when $p \geq 2$ [37]. Instead of completely extracting $q_{i}$ and $\tau_{i}(i=1, \ldots, p)$, which is doubtful as mentioned above and time-consuming, in many applications, it is often useful to determine only the average lifetime which can be expressed in two forms [1]: the intensity-weighted average lifetime

$$
\tau_{I}=\sum_{i=1}^{p} q_{i} \tau_{i}^{2} / \sum_{i=1}^{p} q_{i} \tau_{i}
$$

and the amplitude-weighted average lifetime

$$
\tau_{A}=\sum_{i=1}^{p} q_{i} \tau_{i} / \sum_{i=1}^{p} q_{i}=\sum_{i=1}^{p} q_{i} \tau_{i}
$$

The question about which average lifetime we should use according to the applications has been investigated in [38]. For instance, they suggested:

(a) $\tau_{A}$ can estimate the energy transfer efficiency in FRET [39],

$$
E=1-\frac{I_{D A}}{I_{D}}=1-\frac{\tau_{D A, A}}{\tau_{D, A}},
$$

where $E$ is the energy transfer efficiency, $I_{D A}$ and $I_{D}$ are the fluorescence intensities of the donor in the presence and absence of energy transfer, respectively, and $\tau_{D A, A}$ and $\tau_{D, A}$ are $\tau_{A}$ of the donor in the presence and absence of energy transfer, respectively. $E$ can further estimate the donor-acceptor distance.

(b) $\tau_{A}$ can also assess dynamic quenching behaviors, described by the Stern-Volmer equation [40],

$$
\frac{I_{0}}{I_{1}}=1+K_{D}[Q]=\frac{\tau_{0, A}}{\tau_{1, A}},
$$

where $I_{0}$ and $I_{1}$ are fluorescence intensities, $\tau_{0, A}$ and $\tau_{1, A}$ are $\tau_{A}$ of the fluorophore in the absence and presence of the quencher, respectively, $K_{D}$ is the Stern-Volmer quenching constant, and [Q] is the concentration of the quencher. Additionally, the average radiative rate constant can be expressed as, $k_{r}=Q E / \tau_{1, A}$, where $Q E$ is the quantum yield.

(c) $\tau_{I}$ can be used to estimate the average collisional constant $k_{q}$ from the Stern-Volmer constant $K_{D}$.

Average lifetimes can either be calculated by extracting the lifetime components using model-based LDAs and then using Equations (3) and (4). Or they can be directly obtained with model-free LDAs, such as hardware-friendly center-of-mass methods (CMM) [41-44], the phasor method (Phasor) [45-47], the rapid lifetime determination method (RLD) [30, 48-51], or the integral extraction method (IEM) $[52,53]$, without assuming any decay model.

In this work, we theoretically investigated two types of average lifetimes evaluated by model-free LDAs, examined the performances of $\tau_{A}$ and $\tau_{I}$ estimations using different LDAs, and suggested the choices of LDAs in terms of accuracy, precision, and estimation speeds according to the applications. We also described a multi-exponential decay visualization tool using the ratio $\tau_{A} / \tau_{I}$. Experimental results demonstrate the performance of $\tau_{A} / \tau_{I}$ in comparison with Phasor.

\section{THEORY}

In this section, we derived the average lifetimes determined by the model-free methods, CMM, Phasor, and IEM and described the general work flow of average lifetime estimations with the model-free and model-based LDAs.

As Equation (2), the measured signal $h(t)$ is the convolution of $f(t)$ with $\operatorname{irf}(t)$. Here we focus on the signal $h_{m}$ and $\operatorname{irf} f_{m}$ obtained from a TCSPC system, as shown in Figure 1,

$$
\begin{aligned}
h_{m} & =\sum_{k=0}^{m} \operatorname{irf} f_{k-m} \cdot f_{m}, m=0,1,2, \ldots, M-1, \\
\operatorname{irf} m & =\int_{m \Delta t}^{(m+1) \Delta t} \operatorname{irf}(t) d t \\
f_{m} & =\int_{m \Delta t}^{(m+1) \Delta t} f(t) d t=A \sum_{i=1}^{p} q_{i} \tau_{i} e^{-\frac{m \Delta t}{\tau_{i}}}\left[1-e^{-\frac{\Delta t}{\tau_{i}}}\right],
\end{aligned}
$$

where $h_{m}$ is the photon count collected in Bin $m$ at $t_{m}=(m+1 / 2) \Delta t, M$ is the number of bins, and $\Delta t$ is the time resolution.

(a) CMM 


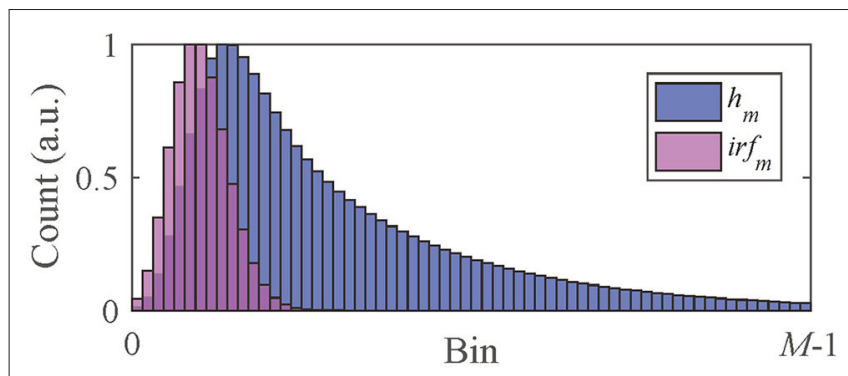

FIGURE 1 | Illustration of $h_{m}$ and $i f_{m}$ obtained with a TCSPC system.

The average lifetime evaluated with CMM is

$$
\begin{aligned}
\tau_{C M M} & =\frac{\int_{0}^{\infty} t \cdot h(t) d t}{\int_{0}^{\infty} h(t) d t}-\frac{\int_{0}^{\infty} t \cdot i r f(t) d t}{\int_{0}^{\infty} i r f(t) d t}=\frac{\sum_{i=1}^{p} q_{i} \tau_{i}^{2}}{\sum_{i=1}^{p} q_{i} \tau_{i}} \\
& \simeq \frac{\sum_{m=0}^{M-1} t_{m} \cdot h_{m}}{\sum_{m=0}^{M-1} h_{m}}-\frac{\sum_{m=0}^{M-1} t_{m} \cdot i r f_{m}}{\sum_{m=0}^{M-1} i r f_{m}}
\end{aligned}
$$

which is equal to $\tau_{I}$. The derivation of Equation (8) is shown in the Appendix.

\section{(b) Phasor}

The average lifetime evaluated with Phasor is

$$
\tau_{P}=\frac{s}{g \omega}=\frac{\sum_{i=1}^{p} q_{i} \tau_{i}^{2} /\left(1+\omega^{2} \tau_{i}^{2}\right)}{\sum_{i=1}^{p} q_{i} \tau_{i} /\left(1+\omega^{2} \tau_{i}^{2}\right)},
$$

where $\omega=2 \pi / T, T=M \Delta t$ is the measurement window, and $g$ and $s$ are the phasor components expressed as

$$
\begin{aligned}
g= & \frac{\int_{0}^{\infty} f(t) \cdot \cos (\omega t) d t}{\int_{0}^{\infty} f(t) d t}=\frac{\sum_{i=1}^{p} q_{i} \tau_{i} /\left(1+\omega^{2} \tau_{i}^{2}\right)}{\sum_{i=1}^{p} q_{i} \tau_{i}} \\
& =\frac{R_{h}+s \cdot I_{i r f}}{R_{i r f}}, \\
s= & \frac{\int_{0}^{\infty} f(t) \cdot \sin (\omega t) d t}{\int_{0}^{\infty} f(t) d t}=\frac{\sum_{i=1}^{p} \omega q_{i} \tau_{i}^{2} /\left(1+\omega^{2} \tau_{i}^{2}\right)}{\sum_{i=1}^{p} q_{i} \tau_{i}} \\
= & \frac{I_{h} \cdot R_{i r f}-R_{h} \cdot I_{i r f}}{R_{i r f}^{2}+I_{i r f}^{2}}
\end{aligned}
$$

where

$$
\begin{aligned}
R_{h} & =\frac{\int_{0}^{\infty} h(t) \cdot \cos (\omega t) d t}{\int_{0}^{\infty} h(t) d t} \simeq \frac{\sum_{m=0}^{M-1} h_{m} \cdot \cos \left(\omega t_{m}\right)}{\sum_{m=0}^{M-1} h_{m}}, \\
I_{h} & =\frac{\int_{0}^{\infty} h(t) \cdot \sin (\omega t) d t}{\int_{0}^{\infty} h(t) d t} \simeq \frac{\sum_{m=0}^{M-1} h_{m} \cdot \sin \left(\omega t_{m}\right)}{\sum_{m=0}^{M-1} h_{m}}, \\
R_{\text {irf }} & =\frac{\int_{0}^{\infty} \operatorname{irf}(t) \cdot \cos (\omega t) d t}{\int_{0}^{\infty} i r f(t) d t} \simeq \frac{\sum_{m=0}^{M-1} i r f_{m} \cdot \cos \left(\omega t_{m}\right)}{\sum_{m=0}^{M-1} \operatorname{irf} m}, \\
I_{i r f} & =\frac{\int_{0}^{\infty} \operatorname{irf}(t) \cdot \sin (\omega t) d t}{\int_{0}^{\infty} \operatorname{irf}(t) d t} \simeq \frac{\sum_{m=0}^{M-1} i r f_{m} \cdot \sin \left(\omega t_{m}\right)}{\sum_{m=0}^{M-1} i r f_{m}} .
\end{aligned}
$$

$\tau_{P}$ is a weighted average lifetime whose weights are $q_{i} \tau_{i} /\left(1+\omega^{2} \tau_{i}^{2}\right)$. If $\tau_{i} \ll T$, then the weights are approximately equal to $q_{i} \tau_{i}$, i.e., $\tau_{P}$ is close to $\tau_{I}$.

\section{(c) IEM}

For IEM, the underlying exponential decay should be extracted by a model-free deconvolution method. With the estimated exponential decay $\hat{f}_{m}$, the average lifetime with IEM is

$$
\begin{aligned}
\tau_{I E M} & =-\frac{\int_{0}^{\infty} g(t) d t}{\int_{0}^{\infty} g^{\prime}(t) d t}=\frac{\sum_{i=1}^{p} q_{i} \tau_{i}}{\sum_{i=1}^{p} q_{i}} \simeq-\frac{\Delta t \sum_{m=0}^{M-1} S_{m} \cdot \hat{f}_{m}}{\hat{f}_{M-1}-\hat{f}_{0}}(10) \\
g(t) & =A \sum_{i=1}^{p} q_{i} \tau_{i} e^{-t / \tau_{i}}\left[1-e^{-\Delta t / \tau_{i}}\right]
\end{aligned}
$$

where $S_{m}=[1 / 3,4 / 3,2 / 3,4 / 3,1 / 3]$ are the coefficients for numerical integration based on Simpson's rule. $\tau_{I E M}$ is actually an estimator for $\tau_{A}$.

Figure 2 summarizes the flow diagram for $\tau_{I}$ and $\tau_{A}$ estimations with different algorithms used in this study. The simulated signals $h_{m}$ and $\operatorname{irf} f_{m}$ are directly sent into CMM and Phasor blocks to estimate $\tau_{I}$. The estimated $f_{m}$ (from $h_{m}$ and $i r f_{m}$ with the Laguerre expansion deconvolution method with $L=16$ and $\alpha=0.912[54,55])$ is sent to IEM to estimate $\tau_{A}$ and sent to the bi-decay center-of-mass method (BCMM; $j=2$ ) [56], the variable projection method (VPM; $j=2$ ) [57], or LSM with a $j$-exponential model (denoted as LSM-j), to estimate $\tau_{I}$ and $\tau_{A}$. CMM and Phasor are fast as no deconvolution routine is needed, whereas IEM, BCMM, VPM, and LSM are direct or iterative estimation approaches once $f_{m}$ is extracted. Artificial neural network assisted analysis tools $[58,59]$ can be included in this diagram, but they are out of the scope of this work.

\section{RESULTS}

\subsection{Simulations}

In reality, it is difficult to characterize a real fluorescence profile with a suitable exponential model described in Equation (1). To demonstrate how model-free analysis can be beneficial, we examined two scenarios. Case A: we used exponential decay signals with $p=1 \sim 4$ to assess the influence of the model mismatch between the signal and the algorithm on $\tau_{I}$ and $\tau_{A}$ estimations. This study is to investigate the scenario when users select a $j$-exponential model to analyze a $p$-exponential decay ( $p$ can be different from $j$ ). Case B: we generated synthetic biexponential $(p=2)$ decay signals to assess the performances of $\tau_{I}$ and $\tau_{A}$ estimations with the model-free and model-based LDAs.

The performances of lifetime estimations can be assessed in two aspects: (1) the accuracy $B_{n}=\left|\hat{\tau}_{n}-\tau_{n}\right| / \tau_{n}$ and the precision $F_{n}=\sqrt{N_{t o t}} \sigma_{\hat{\tau}_{n}} / \hat{\tau}_{n}$ [60], where $n=I$ or $A$ for the intensity- or the amplitude-weighted lifetimes, $\tau_{n}$ and $\hat{\tau}_{n}$ are actual and estimated values, $\sigma_{\hat{\tau}_{n}}$ is the standard deviation of $\hat{\tau}_{n}$, and $N_{\text {tot }}$ is the total photon count. The lower the $F$, the higher the precision $(F=1$ for the ideal case). 


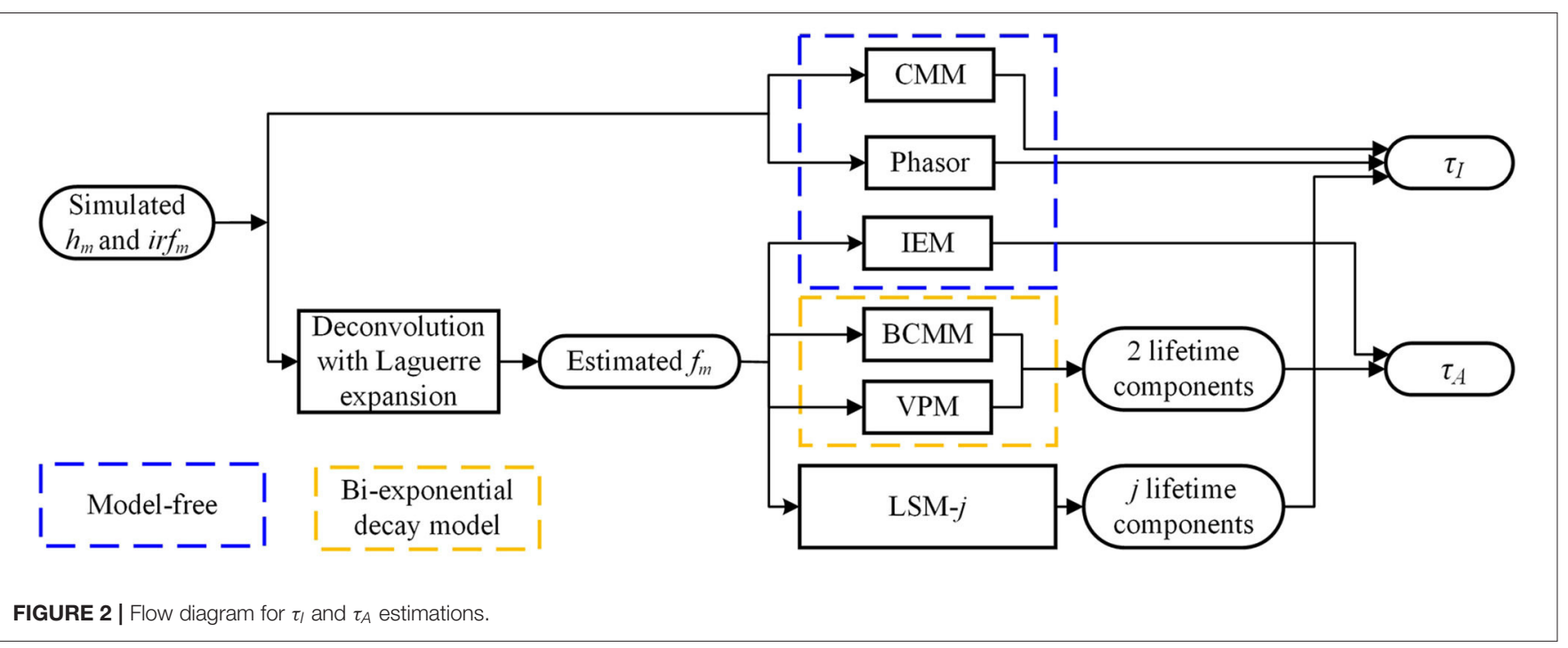

TABLE $1 \mid \tau_{i}, \tau_{l}$, and $\tau_{A}$ for $p=1 \sim 4$ with $q_{i}=1 / p$.

\begin{tabular}{cccc}
\hline $\boldsymbol{p}$ & $\boldsymbol{\tau}_{\boldsymbol{i}}$ (ns) & $\boldsymbol{\tau}_{\boldsymbol{I}}$ (ns) & $\boldsymbol{\tau}_{\boldsymbol{A}}$ (ns) \\
\hline 1 & 2.5 & 2.5 & 2.5 \\
2 & $0.1,2.5$ & 2.40 & 1.3 \\
3 & $0.1,1.3,2.5$ & 2.04 & 1.3 \\
4 & $0.1,0.9,1.7,2.5$ & 1.92 & 1.3 \\
\hline
\end{tabular}

\subsubsection{Case A: Model Mismatch}

Ideally, a bi-exponential signal should be analyzed by a biexponential model. For instance, BCMM, VPM, and LSM-2 are used for bi-exponential decay models, and LSM- $j$ for $j$ exponential models, $j>2$. However, in realistic biological processes, it is difficult to know precisely how many lifetime components a decay profile contains. In traditional FLIM analysis tools, users usually need to select an exponential model to fit measured decays and use the reduced chi-squared to evaluate the goodness-of-fit. If the reduced chi-squared is not satisfactory, then a different exponential model is chosen. This process continues until the reduced chi-squared is acceptable. Often different exponential models can produce similar reduced chisquared values, and the question is which fitting we should use? It is quite common that a $j$-exponential model might analyze a signal containing $p$ lifetime components and $j \neq p$. We would like to know if $p$ is unknown to the user, whether using a different analysis model $(j \neq p)$ would lead to a different biological story.

We generated exponential decay signals $h_{m}(m=0, \ldots, M-1)$ to test the LDAs for $\tau_{I}$ and $\tau_{A}$ estimations. $h_{m}$ can be artificially generated with $f(t)=A \sum_{i=1}^{p} q_{i} \exp \left(-t / \tau_{i}\right)$, where $p=[1,2$, $3,4], q_{i}=1 / p$, and the IRF is approximated with a Poisson distribution $\operatorname{irf} f_{m}=\exp (\lambda) \lambda^{m} / m$ ! with $\lambda=500$ ps, FWHM $\simeq$ $300 \mathrm{ps}$, and $M=256$. The measurement window $T=10 \mathrm{~ns}$, and the total photon count $N_{t o t}=10^{3} . \tau_{i}, \tau_{I}$, and $\tau_{A}$ for each $p$ are summarized in Table 1.

The performances of $\tau_{I}$ and $\tau_{A}$ estimations with the simulated exponential decays are shown in Figures 3A-D for $B_{I}, F_{I}, B_{A}$, and
$F_{A}$, respectively. For model-free LDAs, $B_{I}$ and $B_{A}$ are below $10 \%$ and are independent of $p$. For LSM-1, when $p=1, B_{I}$, and $B_{A}$ are zero, whereas when $p>1, B_{I}$ and $B_{A}$ increase especially for $p=2$. For model-based LDAs where $j>1$, BCMM, VPM, and LSM- $j$ have similar performances even for $p \neq j$, seemingly suggesting that a bi-exponential model can well approximate a signal following an arbitrary $p$-exponential model. We generated 100 signals with $\tau_{i}$ and $q_{i}$ chosen randomly in the ranges of $0.1 \sim 2.5 \mathrm{~ns}$ and $0.1 \sim 0.9$, respectively, for each $p$. BCMM, VPM, and LSM-2 were used to fit the signals with bi-exponential decays. The goodness-of-fit is judged by the reduced chi-squared $\chi^{2}=\frac{1}{M} \sum_{m=0}^{M-1}\left(f_{m}-f_{c, m}\right)^{2} / f_{m}$, where $f_{m}$ and $f_{c, m}$ are actual and fitted signals of Bin $m$. The box plots of $\chi^{2}$ for BCMM, VPM, and LSM-2 are shown in Figures 3E-G, respectively. The $\chi^{2}$ values are insensitive to $p$ for the three LDAs so that we conclude that a bi-exponential decay is suitable to approximate an arbitrary $p$-exponential decay $(p \leq 4)$.

Therefore, if the decay model of the signal is inaccessible, model-free and model-based LDAs, BCMM, VPM, and LSM-2 are enough for $\tau_{I}$ and $\tau_{A}$ estimations.

In practice, users can choose an optimization algorithm and set initial conditions to analyze FLIM images when LSM-2 is used. We would like to know how they can affect $\tau_{I}$ and $\tau_{A}$ estimations. Four bi-exponential decays, Decays $1 \sim 4$, with different parameters $\left(q_{1}, \tau_{1}, \tau_{2}\right)$ were analyzed using LSM-2 with different initial conditions $\left(q_{10}, \tau_{10}, \tau_{20}\right)$, denoted as Init. $1 \sim 4$ listed in Table 2 with $N_{t o t}=10^{3}$. When either of the estimated $\tau_{1}$ and $\tau_{2}$ is larger than $T(10 \mathrm{~ns})$, we say that the estimation fails. The probabilities of producing a failed trial, $P\left(\tau_{1}\right.$ or $\tau_{2}>$ 10 ) and producing biased $\tau_{I}$ and $\tau_{A}$ with $B_{I}$ and $B_{A}>0.3$, i.e., $P\left(B_{n}>0.3\right), n=I$ or $A$, are shown in Figure 4. Figures $4 \mathrm{~A}-\mathrm{F}$ are the LSM-2 results with the unconstrained and constrained trustregion-reflective (TRR) algorithms, respectively. The constraints are $0<q_{1}<1$ and $0<\tau_{1}, \tau_{2}<10$ ns. Figures 4G-I are the LSM-2 results using the Levenberg-Marquardt (LM) algorithm. For the unconstrained TRR, the performances are relatively sensitive to initial conditions. $P\left(\tau_{1}\right.$ or $\left.\tau_{2}>10\right)$ for Init. 4 is quite significant which results in large $P\left(B_{n}>0.3\right), n=I$ 

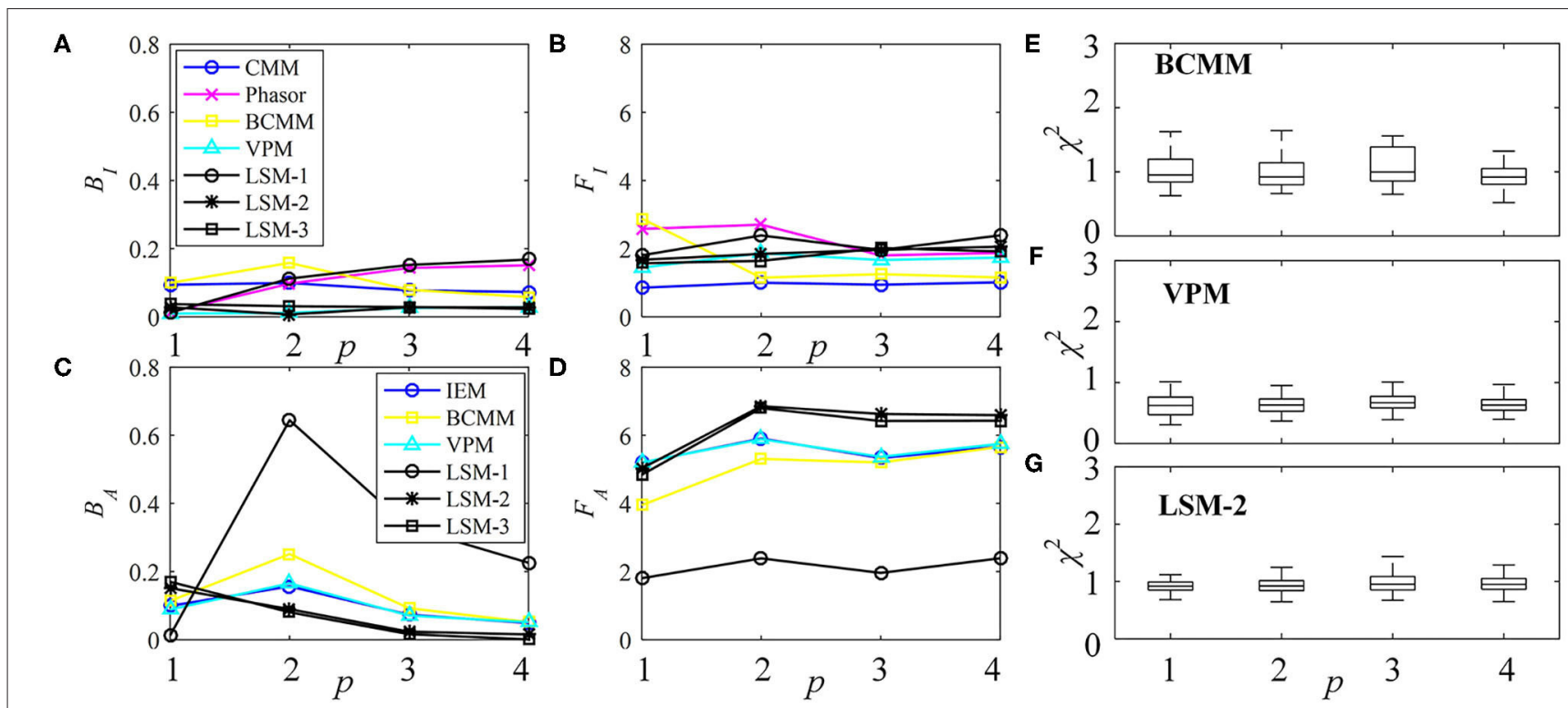

FIGURE $3 \mid$ Performances of $\tau_{l}$ and $\tau_{A}$ estimations with exponential decay signals using different algorithms, (A) $B_{l}$, (B) $F_{l}$, (C) $B_{A}$, and (D) $F_{A}$. (E-G) Box plots of $\chi^{2}$ for BCMM, VPM, and LSM-2.

TABLE 2 | Bi-exponential decays and initial conditions for $\tau_{l}$ and $\tau_{A}$ estimations with LSM-2.

\begin{tabular}{|c|c|c|c|c|c|c|c|}
\hline \multirow[t]{2}{*}{ Decay } & \multicolumn{3}{|c|}{ Parameters } & \multirow[t]{2}{*}{ Init. } & \multicolumn{3}{|c|}{ Initial conditions } \\
\hline & $q_{1}$ & $\tau_{1}$ & $\tau_{2}$ & & $q_{10}$ & $\tau_{10}$ & $\tau_{20}$ \\
\hline 1 & $1 / 2$ & 0.1 & 2.5 & 1 & $1 / 2$ & 0.1 & 0.5 \\
\hline 2 & $4 / 5$ & 0.1 & 2.5 & 2 & $1 / 2$ & 0.1 & 2.5 \\
\hline 3 & $1 / 2$ & 0.5 & 1 & 3 & $1 / 2$ & 2 & 4 \\
\hline 4 & $1 / 2$ & 1 & 2.5 & 4 & $1 / 2$ & 4 & 6 \\
\hline
\end{tabular}

or $A$, for all four decays. Although Init. 3 leads to a low $P$ for Decays $2 \sim 3, P\left(B_{A}>0.3\right)$ for Decay 1 rises to 0.7 . Thus, if the initial conditions are not chosen properly, the quality of $\tau_{I}$ and $\tau_{A}$ images cannot be guaranteed. The constrained TRR and LM are insensitive to initial conditions. Although the LM has failed trials, they barely affect $P\left(B_{n}>0.3\right), n=I$ or $A$. Therefore, to ensure accurate $\tau_{I}$ and $\tau_{A}$ estimations, the constrained TRR and LM are recommended for LSM-2.

\subsubsection{Case B: Performances of Average Lifetime Estimations}

As mentioned above, it might be challenging to use a proper exponential model to describe realistic biological processes; a biexponential model might well approximate them. Here we will use a bi-exponential model to explain why model-free LDAs have the benefits of higher photon efficiency and faster analysis than model-based LDAs for $\tau_{I}$ and $\tau_{A}$ estimations.

$h_{m}$ can be artificially generated with the same IRF used in Case A and $f(t)=A\left[q_{1} \exp \left(-t / \tau_{1}\right)+\left(1-q_{1}\right) \exp \left(-t / \tau_{2}\right)\right]$, where $\tau_{1}<$ $\tau_{2}$ and $q_{1}$ is the amplitude fraction of $\tau_{1}$. Figure $5 \mathbf{A}$ shows the signal and IRF. In FRET and dynamic quenching applications, the fluorescence lifetime of the donor fluorophore is in general decreasing, and we assume $\tau_{2}=2.5 \mathrm{~ns}$ and $\tau_{1}$ varying from 0.1 to $2.5 \mathrm{~ns}$ to emulate FRET or quenching. The theoretical $\tau_{I}, \tau_{A}$, and $\tau_{P}$ with $q_{1}=0.5$ are shown in Figure $5 B$. $\tau_{P}$ has a negative bias from $\tau_{I}$. With $T / \tau_{2}$ increasing, $\tau_{P}$ approaches $\tau_{I}$. Figure $5 \mathbf{B}$ that two different $\left(\tau_{1}, \tau_{2}\right)$ sets can deliver the same $\tau_{I}$, for instance, $(0.32,2.5) \mathrm{ns}$ and $(2.1,2.5) \mathrm{ns}$ have the same $\tau_{I}$ of $2.3 \mathrm{~ns}$.

Therefore, only estimating $\tau_{I}$ can be misleading. Figure 5B also shows that the dynamic range of $\tau_{I}$ is only $2.5-2.23=$ $0.27 \mathrm{~ns}$ and within which the above problem persists. Whereas $\tau_{A}$ does not have this problem for this case. We conducted Monte Carlo simulations to estimate $\tau_{I}$ and $\tau_{A}$ with the simulated signals, including Poisson noise under different conditions $q_{1}=0.2,0.5$, and 0.8 .

The performances of $\tau_{I}$ and $\tau_{A}$ estimations with biexponential decay signals are shown in Figures 6A-D for $B_{I}, F_{I}$, $B_{A}$, and $F_{A}$, respectively. For $\hat{\tau}_{I}, B_{I, C M M}$, and $B_{I, B C M M}$ are roughly 10 and $8 \%$, respectively determined by $T / \tau_{2}$. The larger $T / \tau_{2}$ is, the smaller $B_{I}$ becomes (with $F_{I, C M M}$ and $F_{I, B C M M}$ being closer to 1 ). Phasor has a lower accuracy when $q_{1}$ becomes larger and $\tau_{1}$ smaller, and it is less precise than CMM. VPM and LSM-2 both have a smaller $B_{I}=3 \%$ but higher $F_{I}(1.5 \sim 5)$ than $\mathrm{CMM}$ and BCMM. For $\hat{\tau}_{A}, B_{A}$ is $7 \%$ except for $\tau_{1}=0.1 \mathrm{~ns}$, and $F_{A}$ is around 5 for the four LDAs. Figures 6 C,D show that if only $\tau_{A}$ is needed, there is no need to resort to slower model-based LDAs.

For $\tau_{I}$ estimations, LSM-2 and VPM are preferred when high accuracy is required. Still, they are slower and have lower photon efficiency than CMM and BCMM which means the photon count should be higher to have similar precision, for instance, a relative standard deviation of $5 \%$ can be reached with $N_{\text {tot }}=3,600$ for LSM-2 and $N_{\text {tot }}=500$ for CMM and BCMM. When the 


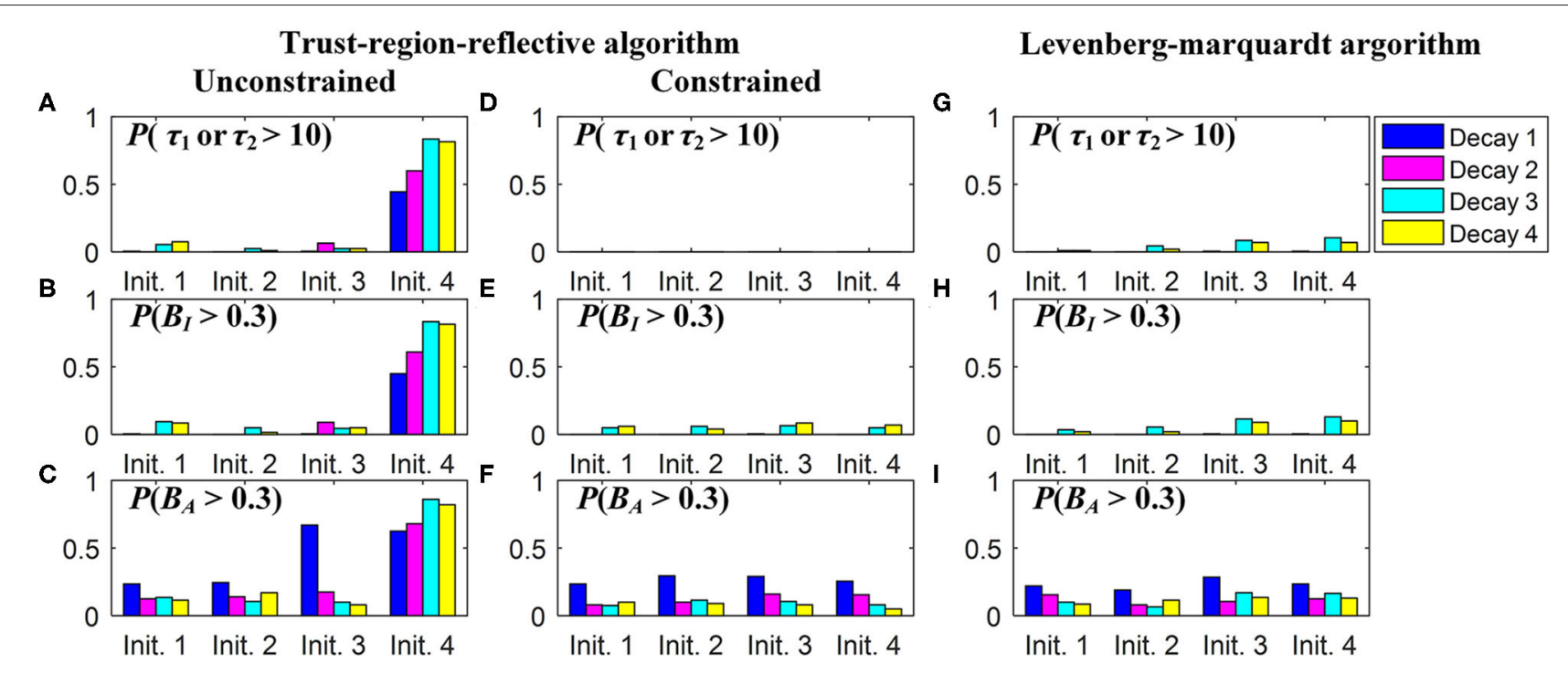

FIGURE $4 \mid(\mathbf{A}, \mathbf{D}, \mathbf{G}) P\left(\tau_{1}\right.$ or $\left.\tau_{2}>10\right),(\mathbf{B}, \mathbf{E}, \mathbf{H}) P\left(B_{l}>0.3\right)$, and $(\mathbf{C}, \mathbf{F}, \mathbf{I}) P\left(B_{A}>0.3\right)$, for estimations of Decays $1 \sim 4$ with Init. $1 \sim 4$ using $(\mathbf{A}-\mathbf{C})$ the unconstrained TRR, (D-F) the constrained TRR, and (G-I) the LM algorithms.

A

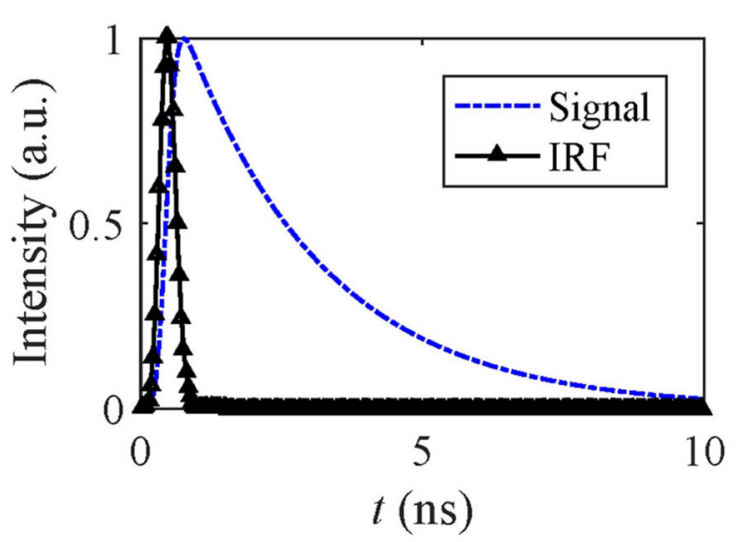

$(0.32,2.5)$

$(2.1,2.5)$

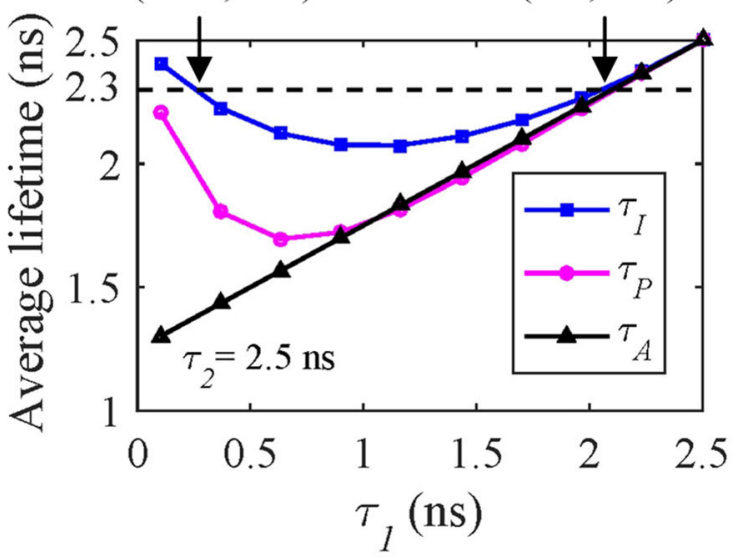

FIGURE 5 | (A) Simulated signal (blue) and IRF (black), (B) theoretical $\tau_{l}$ (blue), $\tau_{A}$ (black), and $\tau_{P}$ (magenta) average lifetimes with $q_{1}=0.5$.

accuracy of CMM or BCMM $\left(10 \% @ T / \tau_{2}=4\right)$ is acceptable, CMM or BCMM should be employed for their high photon efficiency and estimation speeds. CMM is faster than BCMM as it can work without deconvolution. For $\tau_{A}$ estimations, since the performances of IEM, BCMM, VPM, LSM-2 are similar, IEM can be the right candidate for fast analysis. Notice that the $\tau_{A}$ method is less photon efficient than the $\tau_{I}$ method as $F_{A}$ is higher than $F_{I}$.

\subsection{Experimental Results}

tSA201 cells, which are a transformed human kidney cell line, were co-transfected with hP2 $\mathrm{Y}_{12}$-eCFP and hP2 $\mathrm{Y}_{1}$-eYFP receptors. After $48 \mathrm{~h}$ of transfection, the cells on the coverslips were washed once gently with PBS followed by fixation with ice-cold methanol for $10 \mathrm{~min}$ at room temperature. After being washed three times with PBS, they were mounted on to glass microscope slides with Mowiol. The microscope slides were then stored in the dark at room temperature overnight to allow the coverslips to dry, then stored at $4{ }^{\circ} \mathrm{C}$ for later use.

Cells were imaged on LSM510 (Carl Zeiss) equipped with a TCSPC module (SPC-830, Becker \& Hickl GmbH), to determine the fluorescence lifetime and consequently the amount of FRET. The donor is CFP with the excitation wavelength range of $350 \sim 500 \mathrm{~nm}$ and the emission wavelength range of 450 $\sim 600 \mathrm{~nm}$. The acceptor is YFP. The sample is scanned pixel by pixel by a femtosecond Ti:Sapphire laser (Chameleon, 

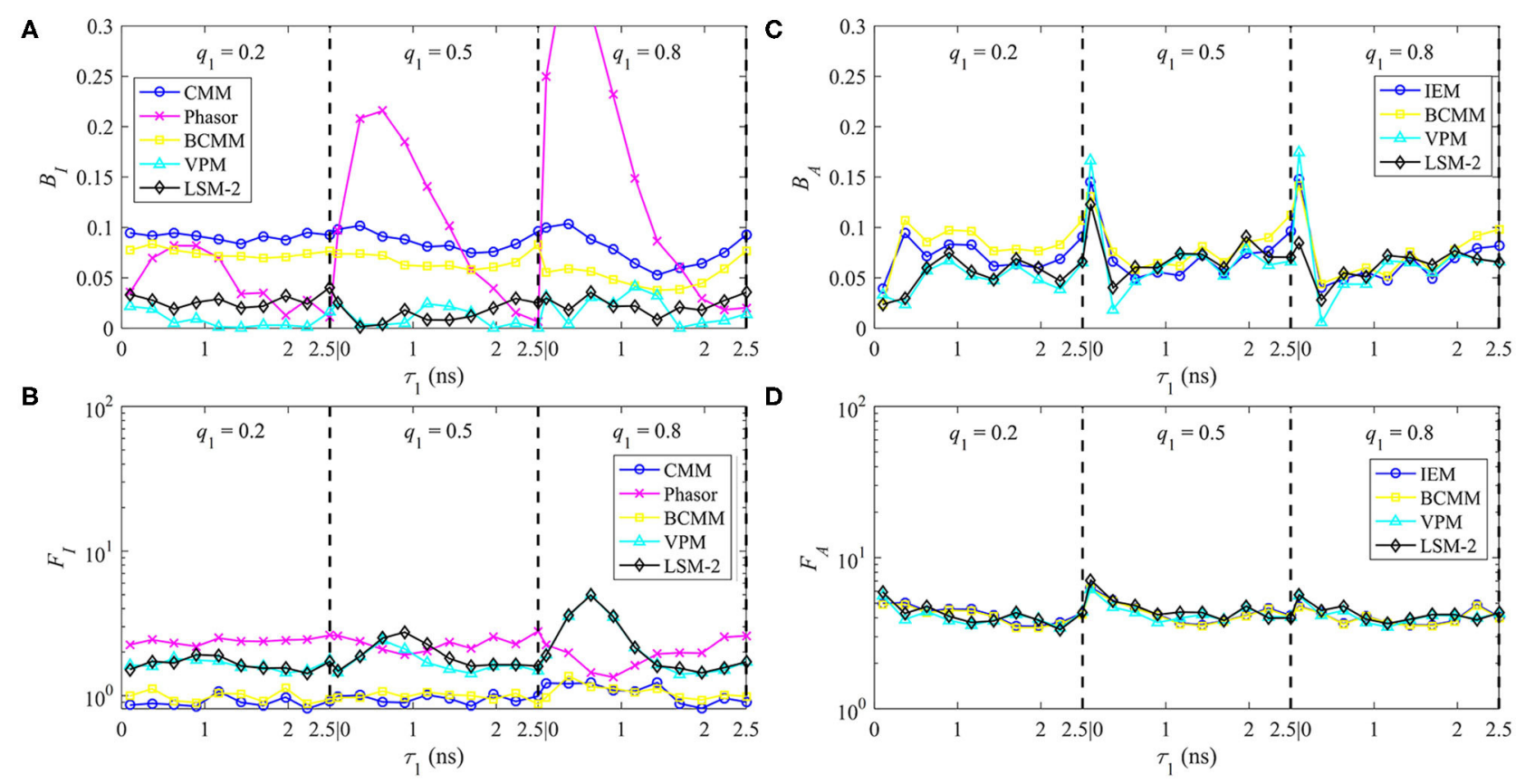

FIGURE 6 | The performances of $\tau_{l}$ and $\tau_{A}$ estimations with bi-exponential decay signals with $\tau_{1}=0.1 \sim 2.5 \mathrm{~ns}, \tau_{2}=2.5 \mathrm{~ns}, q_{1}=0.2,0.5,0.8$, (A) $B_{l}$, (B) $F_{l}$ and $(\mathbf{C})$ $B_{A}$, (D) $F_{A}$.

Coherent) with an average output laser power of $3.8 \mathrm{~W}$ at $800 \mathrm{~nm}$, as a two-photon excitation source to reduce cellular damage. The laser power is controlled with two polarizers. The repetition rate is $80 \mathrm{MHz}$ with illuminating duration $<200 \mathrm{fs}$. The emitted fluorescence signal from the donor is collected through a $63 \times$ water-immersion objective lens (N.A. $=1.0$ ), a $480 \sim 520 \mathrm{~nm}$ bandpass filter, and transferred into a photomultiplier tube (PMT) detector. The FLIM scanning was performed in a dark room containing the microscope. A set of experimental data $(256 \times 256$ pixels, $M=256, T=10 \mathrm{~ns})$ was collected over an exposure period of up to $15 \mathrm{~min}$. The IRF is obtained from the measurement of dried urea $\left[\left(\mathrm{NH}_{2}\right)_{2} \mathrm{CO}\right]$ [61].

\subsubsection{Average Lifetime Images With LSM, CMM, and IEM}

Figures 7A,C show the $\tau_{I}$ and $\tau_{A}$ images of the data evaluated by LSM-2 with an execution time of $410 \mathrm{~s}$. The lifetime images were evaluated on Matlab R2016a, 64-bit with the Intel(R) Celeron(R) CPU (2950M @ $2 \mathrm{GHz}$ ) with 20923 pixels above an intensity threshold. Figures 7B,D show the $\tau_{I}$ and $\tau_{A}$ images evaluated by CMM and IEM with execution times of 0.25 and $92.3 \mathrm{~s}$, respectively. IEM can be further accelerated to $0.6 \mathrm{~s}$ per image with histogram classification methods (we will report the details soon), as shown in Figure 7E. Although Fast-IEM causes a small bias in some pixels, the mean square error is acceptable with 0.005 $\mathrm{ns}^{2}$. The color bar represents lifetimes and the pixel brightness represents photon counts. The Figures $7 \mathbf{F}, \mathbf{G}$ are histograms of $\tau_{I}$ and $\tau_{A}$, respectively. Although the histogram of $\tau_{I}$ with CMM deviates slightly from the one with LSM-2, CMM is 1,800 -fold faster than LSM-2. If $T / \tau_{i}>4$, the bias of $\tau_{I}$ with CMM would become smaller. The $\tau_{A}$ images are almost the same with IEM and LSM-2, whereas IEM and Fast-IEM are much faster than LSM-2.

Since the FRET efficiency $E$ has a linear relationship with the average lifetimes as shown in Equation (5), Figures 7A-E can also be used to represent $E$ images with the color bar in the range of 0 $\sim 100 \%$. As we mentioned in Introduction, it is straightforward to obtain $E$ images from $\tau_{A}$ images, so that Figures $7 \mathbf{C}-\mathbf{E}$ are proper $E$ images. If $\tau_{I}$ images are misused for $E$, the results would be different, as shown in Figures 7A,B, leading to a different biological story.

\subsubsection{Visualization of Multi-Exponential Decays With $\tau_{A} / \tau_{I}$}

$\tau_{I}$ and $\tau_{A}$ can not only access the essential parameters in FRET and dynamic quenching processes but also indicate the positions where multi-exponential decays occur. As mentioned previously, a fluorescence signal can be approximated by a bi-exponential decay, so that the ratio of $\tau_{I}$ and $\tau_{A}$ can be expressed as

$$
\frac{\tau_{A}}{\tau_{I}}=\frac{\left[1+q_{1}(R-1)\right]^{2}}{1+q_{1}\left(R^{2}-1\right)},
$$

where $R=\tau_{1} / \tau_{2}$. The distribution of $\tau_{A} / \tau_{I}$ (Figure 8) shows that when $R \simeq 1$ or $q_{1} \simeq 0$ or $1, \tau_{A} / \tau_{I} \simeq 1$. With a decrease of $R$ or an increase of $q_{1}, \tau_{A} / \tau_{I}$ decreases. Therefore, the ranges of $q_{1}$ and $R$ of a pixel can be determined by $\tau_{A} / \tau_{I}$.

To present the multi-exponential decay visualization performance of $\tau_{A} / \tau_{I}$, the $\tau_{I}$ and $\tau_{A}$ images evaluated by LSM-2, 
A

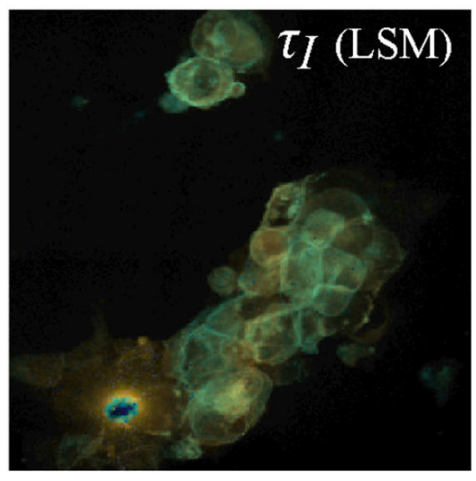

C

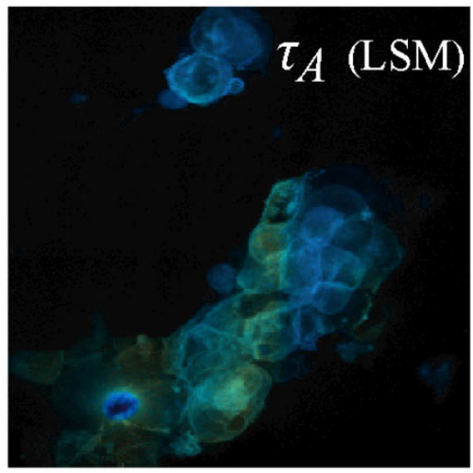

$\mathbf{F}$

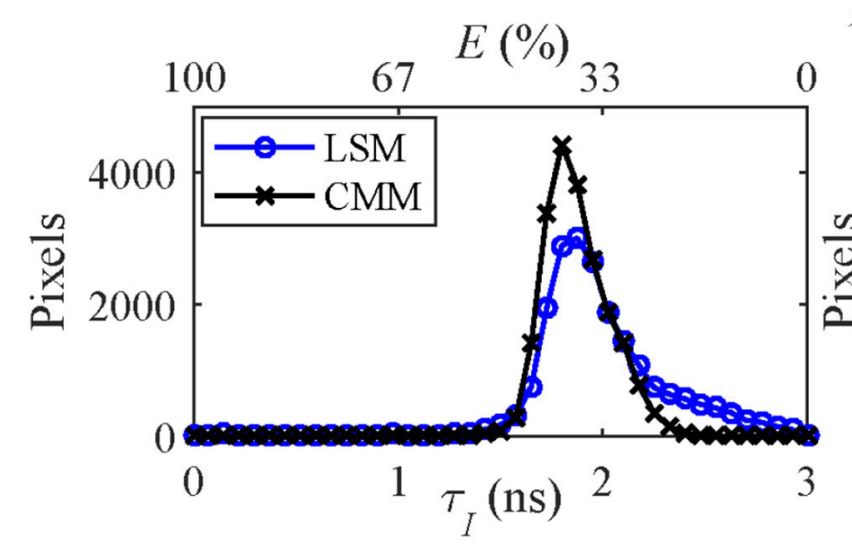

B

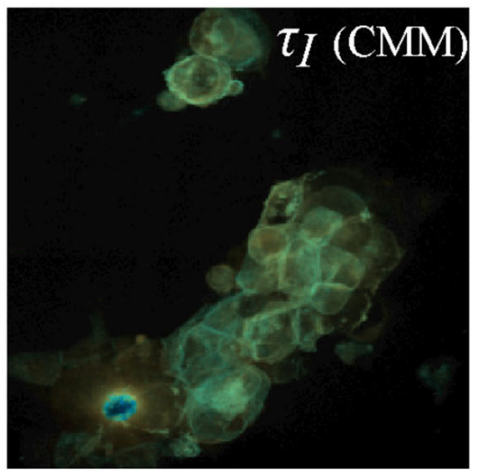

D

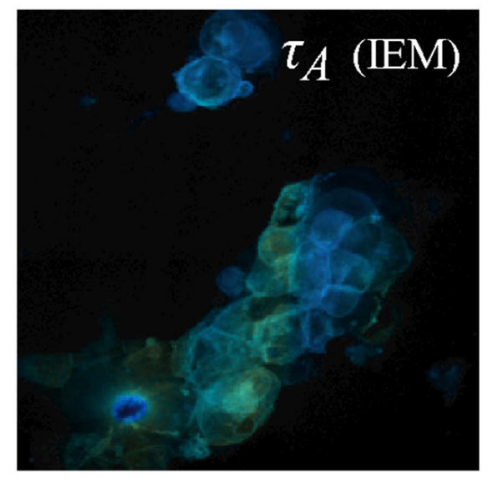

G
$\tau_{I}$ or $\tau_{A}(\mathrm{~ns}) \mid E(\%)$

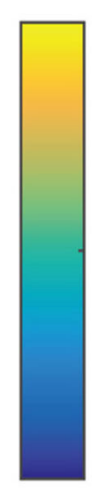

3

0

E

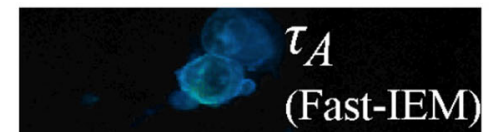

FIGURE $7 \mid(\mathbf{A}, \mathbf{C}) \tau_{l}$ and $\tau_{A}$ images evaluated with LSM-2, (B,D) $\tau_{l}$ and $\tau_{A}$ images evaluated with CMM and IEM, respectively. (E) $\tau_{A}$ image with Fast-IEM. (F) Histograms of $\tau_{\text {I }}$ with LSM-2 (blue) and CMM (black). (G) Histograms of $\tau_{A}$ with LSM-2 (blue), IEM (magenta), and Fast-IEM (yellow). The color bar represents lifetimes and the pixel brightness represents photon counts. (A-E) Can also represent FRET efficiency $(E)$ images evaluated with the corresponding lifetime images with the color bar representing the range of $E, 0 \sim 100 \%$. (F,G) Can also be used to show the histograms of $E$ with the upper $x$ label.

Figures 9A,B, were used to generate the $\tau_{A} / \tau_{I}$ image as shown in Figure 9C. The histograms of $\tau_{I}$ and $\tau_{A}$ and the phasor plot are shown in Figures 9D,E. Figure 9F shows the possible range of $q_{1}$ and $R$ of the selected pixels in Figure 9C. Figures 9C,F share the same color bar. Figure $9 D$ shows that $\tau_{A}$ has a broader lifetime dynamic range than $\tau_{I}$, which is consistent with the theoretical lines shown in Figure 5B. The $\tau_{A}$ histogram shows two clusters with different peaks, whereas the $\tau_{I}$ histogram only indicates a single merged group, meaning that there is no way to differentiate these two clusters. It is why using $\tau_{I}$ to analyze samples with a strong FRET can be misleading.

The results of the selected pixels within different $\tau_{A} / \tau_{I}$ ranges are shown in Figure 10, $\tau_{A} / \tau_{I}=0.2 \sim 0.5$, and Figure 11, $\tau_{A} / \tau_{I}$ $=0.5 \sim 1$. For the pixels with $\tau_{A} / \tau_{I}=0.2 \sim 0.5$, the histograms clearly show that $\tau_{A}$ is much smaller than $\tau_{I}$, which means the difference between $\tau_{1}$ and $\tau_{2}$ is significant. Figure 10F shows that 
the ranges of $q_{1}$ and $R$ are approximately $0.5 \sim 1$ and $0 \sim 0.2$, respectively. For the pixels with $\tau_{A} / \tau_{I}=0.5 \sim 1, \tau_{A}$ is closer to $\tau_{I}$, meaning the pixels have decays close to mono-exponential. Separating the average lifetime images with $\tau_{A} / \tau_{I}$ is easier than

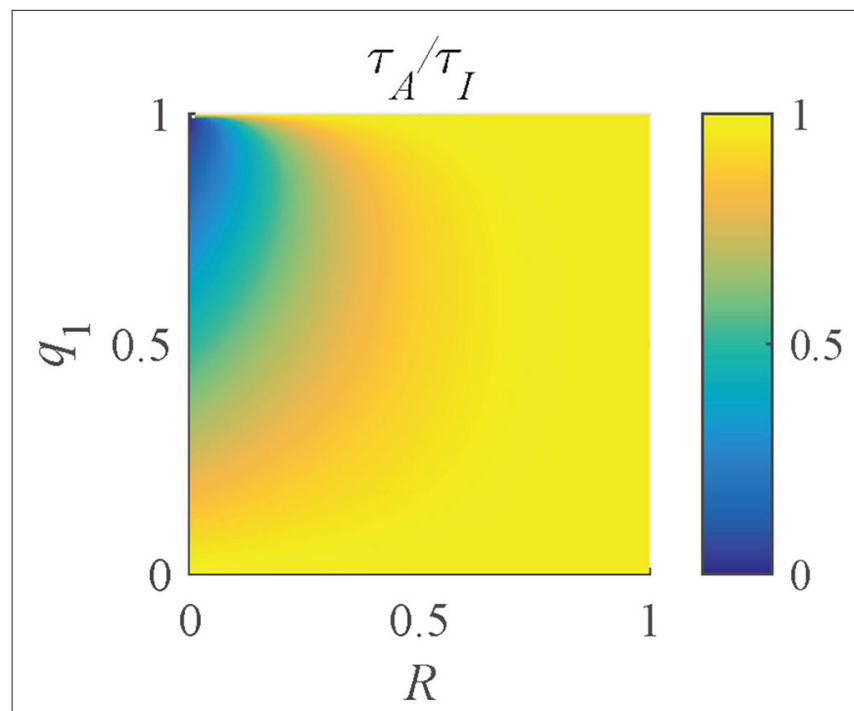

FIGURE 8 | Distribution of $\tau_{A} / \tau_{\text {l }}$ with $q_{1}=0 \sim 1$ and $R=0 \sim 1$. phasor plots because $\tau_{A} / \tau_{I}$ is one dimensional and phasors are two dimensional. Furthermore, $\tau_{A} / \tau_{I}$ can show the $q_{1}$ and $R$ ranges more intuitively than phasor plots. $\tau_{A} / \tau_{I}$ can be a useful tool to visualize the properties of the fluorescence decays within a lifetime image.

\section{DISCUSSION}

In realistic samples, fluorescence signals always follow multiexponential decay models. However, extracting lifetime components with a traditional fitting method is a timeconsuming process. For some applications that require calculating FRET efficiency and accessing dynamic quenching behaviors, average lifetimes are satisfactory. Model-free lifetime determination algorithms can be used to evaluate average lifetimes directly, for instance, CMM and Phasor for intensityweighted average lifetimes $\tau_{I}$ and IEM for amplitude-weighted average lifetimes $\tau_{A}$. Discussions of the influence of the model mismatch between the real signal and the model-based LDAs on $\tau_{I}$ and $\tau_{A}$ estimations suggest that a bi-exponential model can well-approximate a signal following a multiple-exponential model. The results of the Monte-Carlo simulations suggest that VPM and LSM based on a bi-exponential model can be used for applications requiring high accuracy. The constrained TRR and LM algorithms with proper initial conditions are supported for LSM to guarantee accuracy. In contrast, CMM and IEM are recommended for applications requiring high estimation speeds.

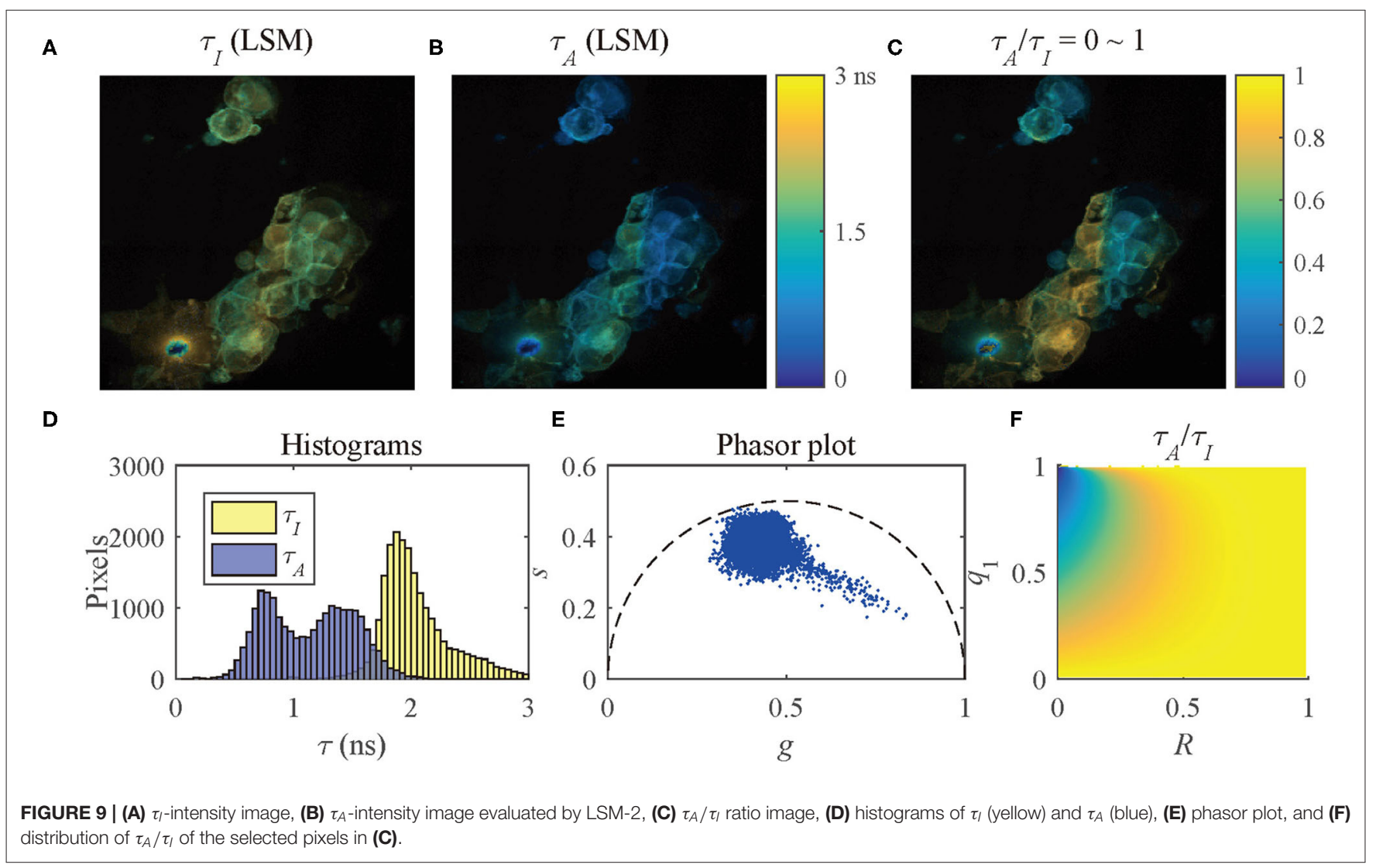



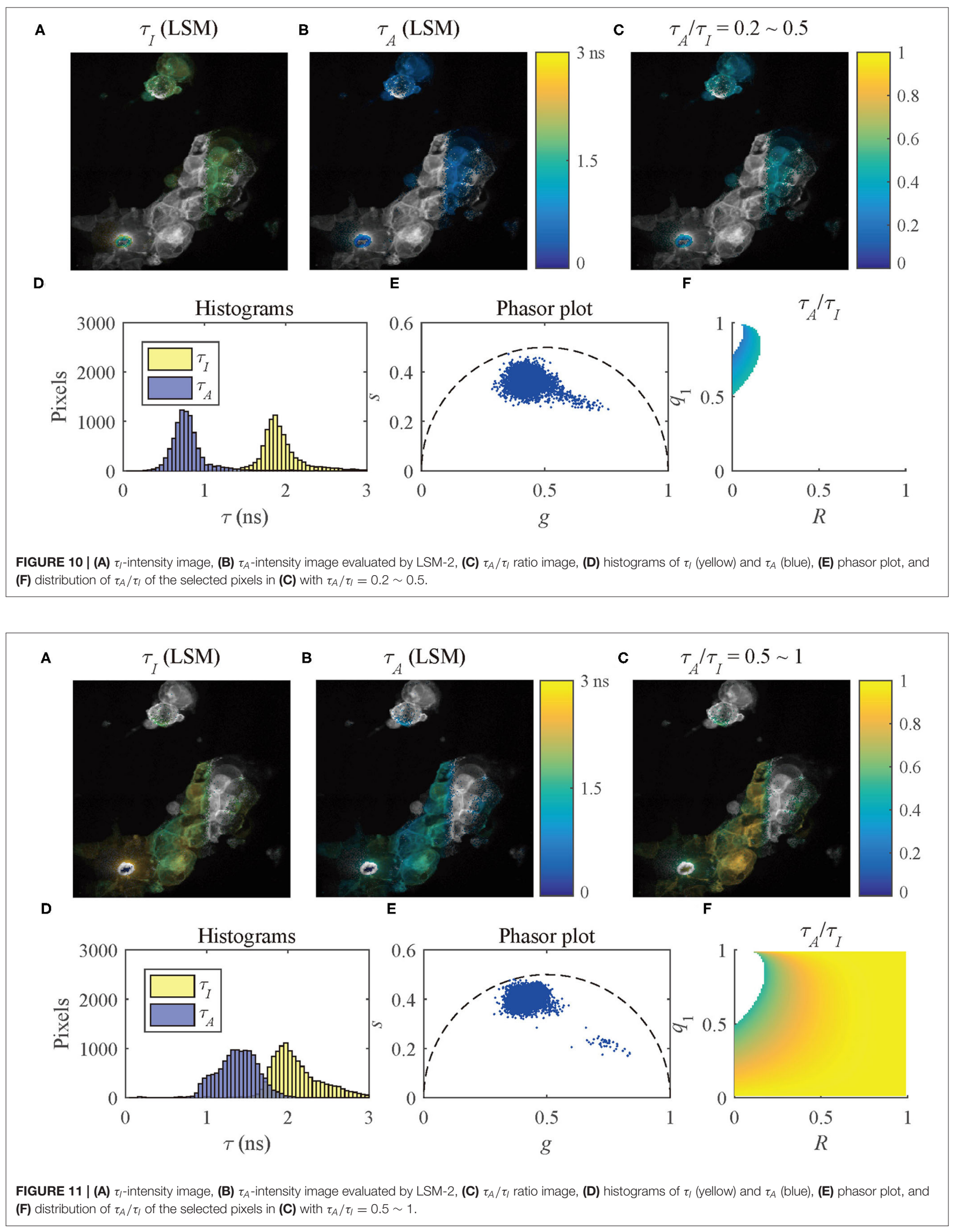
We also explained why $\tau_{I}$ models can be misleading, and $\tau_{I}$ and $\tau_{A}$ models should be considered. Experimental data were used to compare the performances of LSM-2, CMM, and IEM for evaluating $\tau_{I}$ and $\tau_{A}$ images. Similar $\tau_{I}$ and $\tau_{A}$ images were generated, whereas CMM and IEM are much faster than LSM-2. The data were further analyzed with $\tau_{A} / \tau_{I}$, which is capable of indicating the possible ranges of the amplitude proportion of the short lifetime and the ratio of the short and long lifetimes. We believe $\tau_{A} / \tau_{I}$ is a useful and intuitive tool for visualizing multi-exponential decays in a lifetime image.

\section{DATA AVAILABILITY STATEMENT}

FLIM image raw data and the instrument response are available at https://doi.org/10.15129/062e9e11-9d7f-49e5-a3322b10d695bdcd.

\section{REFERENCES}

1. Lakowicz JR. Principles of Fluorescence Spectroscopy. New York, NY: Springer (2006). doi: 10.1007/978-0-387-46312-4

2. Berezin MY, Achilefu S. Fluorescence lifetime measurements and biological imaging. Chem Rev. (2010) 110:2641-84. doi: 10.1021/cr900343z

3. Ning Y, Cheng S, Wang JX, Liu YW, Feng W, Li F, et al. Fluorescence lifetime imaging of upper gastrointestinal $\mathrm{pH}$ in vivo with a lanthanide based nearinfrared $\tau$ probe. Chem Sci. (2019) 10:4227-35. doi: 10.1039/C9SC00220K

4. Agronskaia AV, Tertoolen L, Gerritsen HC. Fast fluorescence lifetime imaging of calcium in living cells. J Biomed Opt. (2004) 9:1230-7. doi: $10.1117 / 1.1806472$

5. Zheng K, Jensen TP, Rusakov DA. Monitoring intracellular nanomolar calcium using fluorescence lifetime imaging. Nat Protoc. (2018) 13:581-97. doi: $10.1038 /$ nprot.2017.154

6. Hosny NA, Lee DA, Knight MM. Single photon counting fluorescence lifetime detection of pericellular oxygen concentrations. J Biomed Opt. (2012) 17:016007. doi: 10.1117/1.JBO.17.1. 016007

7. Peng X, Yang Z, Wang J, Fan J, He Y, Song F, et al. Fluorescence ratiometry and fluorescence lifetime imaging: using a single molecular sensor for dual mode imaging of cellular viscosity. J Am Chem Soc. (2011) 133:6626-35. doi: 10.1021/ja1104014

8. Okabe K, Inada N, Gota C, Harada Y, Funatsu T, Uchiyama S. Intracellular temperature mapping with a fluorescent polymeric thermometer and fluorescence lifetime imaging microscopy. Nat Commun. (2012) 3:705. doi: $10.1038 /$ ncomms 1714

9. Fruhwirth GO, Fernandes LP, Weitsman G, Patel G, Kelleher M, Lawler $\mathrm{K}$, et al. How förster resonance energy transfer imaging improves the understanding of protein interaction networks in cancer biology. ChemPhysChem. (2011) 12:442-61. doi: 10.1002/cphc.201000866

10. Marcu L. Fluorescence lifetime techniques in medical applications. Ann Biomed Eng. (2012) 40:304-31. doi: 10.1007/s10439-011-0495-y

11. Nobis M, McGhee EJ, Morton JP, Schwarz JP, Karim SA, Quinn J, et al. Intravital FLIM-FRET imaging reveals Dasatinib-induced spatial control of Src in pancreatic cancer. Cancer Res. (2013) 73:4674-86. doi: 10.1158/0008-5472.CAN-12-4545

12. Levitt JA, Poland SP, Krstajic N, Pfisterer K, Erdogan A, Barber PR, et al. Quantitative real-time imaging of intracellular FRET biosensor dynamics using rapid multi-beam confocal FLIM. Sci Rep. (2020) 10:5146. doi: 10.1038/s41598-020-61478-1

13. Becker W. Fluorescence lifetime imaging-applications and instrumental principles. In: Bradshaw RA, Stahl PD, editors. Encyclopedia of Cell Biology. Vol. 2. Waltham, MA: Elsevier. (2016). p. 107-20. doi: 10.1016/B978-0-12-394447-4.20095-3

14. Coda S, Thompson AJ, Kennedy GT, Roche KL, Ayaru L, Bansi DS, et al. Fluorescence lifetime spectroscopy of tissue autofluorescence in normal and

\section{AUTHOR CONTRIBUTIONS}

YL conducted theoretical and experimental analysis and developed analysis tools. MS and MC conceived FRET experiments and prepared samples. SN and YC contributed to FRET-FLIM experiments. JT contributed to tool developments. DL initiated the research concept and supervised the project. All authors wrote and revised the paper.

\section{FUNDING}

We would like to acknowledge support from Medical Research Scotland, China Scholarship Council, and Engineering and Physical Sciences Research Council (EP/M506643/1).

diseased colon measured ex vivo using a fiber-optic probe. Biomed Opt Express. (2014) 5:515. doi: 10.1364/BOE.5.000515

15. Canals J, Franch N, Alonso O, Vilá A, Diéguez A. A point-of-care device for molecular diagnosis based on CMOS SPAD detectors with integrated microfluidics. Sensors. (2019) 19:445. doi: 10.3390/s19030445

16. Alonso O, Franch N, Canals J, Arias-Alpízar K, de la Serna E, Baldrich E, et al. An internet of things-based intensity and time-resolved fluorescence reader for point-of-care testing. Biosens Bioelectron. (2020) 154:112074. doi: 10.1016/j.bios.2020.112074

17. Heger Z, Kominkova M, Cernei N, Krejcova L, Kopel P, Zitka O, et al. Fluorescence resonance energy transfer between green fluorescent protein and doxorubicin enabled by DNA nanotechnology. Electrophoresis. (2014) 35:3290-301. doi: 10.1002/elps.201400166

18. Karpf S, Riche CT, Di Carlo D, Goel A, Zeiger WA, Suresh A, et al. Spectro-temporal encoded multiphoton microscopy and fluorescence lifetime imaging at kilohertz frame-rates. Nat Commun. (2020) 11:2062. doi: 10.1038/s41467-020-15618-w

19. Hirmiz N, Tsikouras A, Osterlund EJ, Richards M, Andrews DW, Fang Q. Highly multiplexed confocal fluorescence lifetime microscope designed for screening applications. IEEE J Select Top Quant Electron. (2020) 27:1-9. doi: 10.1109/JSTQE.2020.2997834

20. Ehn A, Johansson O, Arvidsson A, Aldén M, Bood J. Single-laser shot fluorescence lifetime imaging on the nanosecond timescale using a Dual Image and Modeling Evaluation algorithm. Opt Exp. (2012) 20:3043. doi: 10.1364/OE.20.003043

21. Jonsson M, Ehn A, Christensen M, Aldén M, Bood J. Simultaneous one-dimensional fluorescence lifetime measurements of $\mathrm{OH}$ and $\mathrm{CO}$ in premixed flames. Appl Phys B. (2014) 115:35-43. doi: 10.1007/s00340-0135570-7

22. Ehn A, Zhu J, Li X, Kiefer J. Advanced laser-based techniques for gas-phase diagnostics in combustion and aerospace engineering. Appl Spectrosc. (2017) 71:341-66. doi: 10.1177/0003702817690161

23. Becker W. Advanced Time-Correlated Single Photon Counting Techniques. Heidelberg: Springer (2005). doi: 10.1007/3-540-28882-1

24. Rinnenthal JL, Börnchen C, Radbruch H, Andresen V, Mossakowski A, Siffrin $\mathrm{V}$, et al. Parallelized TCSPC for dynamic intravital fluorescence lifetime imaging: quantifying neuronal dysfunction in neuroinflammation. PLoS ONE. (2013) 8:e60100. doi: 10.1371/journal.pone.0060100

25. Erdogan AT, Finlayson N, Williams GS, Williams E, Henderson RK. Video rate spectral fluorescence lifetime imaging with a 512 x 16 SPAD line sensor. In: Goda K, Tsia KK, editors. High-Speed Biomedical Imaging and Spectroscopy IV. San Francisco, CA: SPIE (2019). p. 22. doi: 10.1117/12.2509607

26. Cole MJ, Siegel J, Webb SED, Jones R, Dowling K, Dayel MJ, et al. Timedomain whole-field fluorescence lifetime imaging with optical sectioning. $J$ Microsc. (2001) 203:246-57. doi: 10.1046/j.1365-2818.2001.00894.x

27. Grant DM, McGinty J, McGhee EJ, Bunney TD, Owen DM, Talbot $\mathrm{CB}$, et al. High speed optically sectioned fluorescence lifetime imaging 
permits study of live cell signaling events. Opt Exp. (2007) 15:15656. doi: 10.1364/OE.15.015656

28. Gu L, Hall DJ, Qin Z, Anglin E, Joo J, Mooney DJ, et al. In vivo timegated fluorescence imaging with biodegradable luminescent porous silicon nanoparticles. Nat Commun. (2013) 4:2326. doi: 10.1038/ncomms3326

29. Camborde L, Jauneau A, Briére C, Deslandes L, Dumas B, Gaulin E. Detection of nucleic acid-protein interactions in plant leaves using fluorescence lifetime imaging microscopy. Nat Protoc. (2017) 12:1933-50. doi: 10.1038/nprot.2017.076

30. Li DDU, Ameer-Beg S, Arlt J, Tyndall D, Walker R, Matthews DR, et al. TimeDomain fluorescence lifetime imaging techniques suitable for solid-state imaging sensor arrays. Sensors. (2012) 12:5650-69. doi: 10.3390/s120505650

31. Bajzer Ž, Prendergast FG. [10] Maximum likelihood analysis of fluorescence data. Methods Enzymol. (1992) 210:200-37. doi: 10.1016/0076-6879(92)10012-3

32. Verveer PJ, Hanley QS. Chapter 2: Frequency domain FLIM theory, instrumentation, and analysis. In: Gadella TWJ, editor. Laboratory Techniques in Biochemistry and Molecular Biology. New York, NY: Elesvier B.V. (2009). p. 59-94. doi: 10.1016/S0075-7535(08)00002-8

33. Zhao Q, Schelen B, Schouten R, van den Oever R, Leenen R, van Kuijk H, et al. Modulated electron-multiplied fluorescence lifetime imaging microscope: allsolid-state camera for fluorescence lifetime imaging. J Biomed Opt. (2012) 17:126020. doi: 10.1117/1.JBO.17.12.126020

34. Howard SS, Straub A, Horton NG, Kobat D, Xu C. Frequency-multiplexed in vivo multiphoton phosphorescence lifetime microscopy. Nat Photon. (2013) 7:33-7. doi: 10.1038/nphoton.2012.307

35. Serafino MJ, Applegate BE, Jo JA. Direct frequency domain fluorescence lifetime imaging using field programmable gate arrays for real time processing. Rev Sci Instrum. (2020) 91:033708. doi: 10.1063/1.5127297

36. James F. Statistical Methods in Experimental Physics. Singapore: World Scientific (2008).

37. Fišerová $\mathrm{E}$, Kubala $\mathrm{M}$. Mean fluorescence lifetime and its error. J Luminesc. (2012) 132:2059-64. doi: 10.1016/j.jlumin.2012.03.038

38. Sillen A, Engelborghs Y. The correct use of "average" fluorescence parameters. Photochem Photobiol. (1998) 67:475-486. doi: 10.1111/j.1751-1097.1998.tb09082.x

39. Gadella T. FRET and FLIM Techniques. Amsterdam: Elsevier B.V. (2009).

40. Gehlen MH. The centenary of the Stern-Volmer equation of fluorescence quenching: from the single line plot to the SV quenching map. J Photochem Photobiol C. (2020) 42:100338. doi: 10.1016/j.jphotochemrev.2019.100338

41. Li DDU, Arlt J, Tyndall D, Walker R, Richardson J, Stoppa D, et al. Video-rate fluorescence lifetime imaging camera with CMOS single-photon avalanche diode arrays and high-speed imaging algorithm. J Biomed Opt. (2011) 16:096012. doi: 10.1117/1.3625288

42. Padilla-Parra S, Auduge N, Coppey-Moisan M, Tramier M. Non fitting based FRET-FLIM analysis approaches applied to quantify protein-protein interactions in live cells. Biophys Rev. (2011) 3:63-70. doi: 10.1007/s12551-011-0047-6

43. Won Y, Moon S, Yang W, Kim D, Han WT, Kim DY. High-speed confocal fluorescence lifetime imaging microscopy (FLIM) with the analog mean delay (AMD) method. Opt Exp. (2011) 19:3396. doi: 10.1364/OE.19. 003396

44. Hwang W, Kim D, Lee S, Won YJ, Moon S, Kim DY. Analysis of biexponential decay signals in the analog mean-delay fluorescence lifetime measurement method. Opt Commun. (2019) 443:136-43. doi: 10.1016/j.optcom.2019.02.059

45. Digman MA, Caiolfa VR, Zamai M, Gratton E. The phasor approach to fluorescence lifetime imaging analysis. Biophys J. (2008) 94:L14-6. doi: 10.1529/biophysj.107.120154

46. Stringari C, Cinquin A, Cinquin O, Digman MA, Donovan PJ, Gratton E. Phasor approach to fluorescence lifetime microscopy distinguishes different metabolic states of germ cells in a live tissue. Proc Natl Acad Sci USA. (2011) 108:13582-7. doi: 10.1073/pnas.1108161108
47. Ranjit S, Malacrida L, Jameson DM, Gratton E. Fit-free analysis of fluorescence lifetime imaging data using the phasor approach. Nat Protoc. (2018) 13:1979-2004. doi: 10.1038/s41596-018-0026-5

48. Ballew RM, Demas JN. An error analysis of the rapid lifetime determination method for the evaluation of single exponential decays. Analyt Chem. (1989) 61:30-3. doi: 10.1021/ac00176a007

49. Elangovan M, Day RN, Periasamy A. Nanosecond fluorescence resonance energy transfer-fluorescence lifetime imaging microscopy to localize the protein interactions in a single living cell. J Microsc. (2002) 205:3-14. doi: 10.1046/j.0022-2720.2001.00984.x

50. Moore C, Chan SP, Demas JN, DeGraff BA. Comparison of methods for rapid evaluation of lifetimes of exponential decays. Appl Spectrosc. (2004) 58:603-7. doi: 10.1366/000370204774103444

51. Elson DS, Munro I, Requejo-Isidro J, McGinty J, Dunsby C, Galletly N, et al. Real-time time-domain fluorescence lifetime imaging including single-shot acquisition with a segmented optical image intensifier. New J Phys. (2004) 6:180. doi: 10.1088/1367-2630/6/1/180

52. Li DDU, Bonnist E, Renshaw D, Henderson R. On-chip, time-correlated, fluorescence lifetime extraction algorithms and error analysis. J Opt Soc Am A. (2008) 25:1190. doi: 10.1364/JOSAA.25.001190

53. Li DDU, Arlt J, Richardson J, Walker R, Buts A, Stoppa D, et al. Real-time fluorescence lifetime imaging system with a $32 \times 320.13 \mu \mathrm{m}$ CMOS low dark-count single-photon avalanche diode array. Opt Exp. (2010) 18:10257. doi: 10.1364/OE.18.010257

54. Liu J, Sun Y, Qi J, Marcu L. A novel method for fast and robust estimation of fluorescence decay dynamics using constrained least-squares deconvolution with Laguerre expansion. Phys Med Biol. (2012) 57:843-65. doi: 10.1088/0031-9155/57/4/843

55. Zhang Y, Chen Y, Li DDU. Optimizing Laguerre expansion based deconvolution methods for analysing bi-exponential fluorescence lifetime images. Opt Exp. (2016) 24:13894. doi: 10.1364/OE.24.013894

56. Li DDU, Yu H, Chen Y. Fast bi-exponential fluorescence lifetime imaging analysis methods. Opt Lett. (2015) 40:336-9. doi: 10.1364/OL.40.000336

57. Zhang Y, Cuyt A, Lee W, Lo Bianco G, Wu G, Chen Y, et al. Towards unsupervised fluorescence lifetime imaging using low dimensional variable projection. Opt Exp. (2016) 24:26777. doi: 10.1364/OE.24.026777

58. Wu G, Nowotny T, Zhang Y, Yu HQ, Li DDU. Artificial neural network approaches for fluorescence lifetime imaging techniques. Opt Lett. (2016) 41:2561. doi: 10.1364/OL.41.002561

59. Smith JT, Yao R, Sinsuebphon N, Rudkouskaya A, Un N, Mazurkiewicz J, et al. Fast fit-free analysis of fluorescence lifetime imaging via deep learning. Proc Natl Acad Sci USA. (2019) 116:24019-30. doi: 10.1073/pnas.1912707116

60. Draaijer A, Sanders R, Gerritsen HC. Fluorescence lifetime imaging, a new tool in confocal microscopy. In: Pawley JB, editor. Handbook of Biological Confocal Microscopy. Boston, MA: Springer US (1995). p. 491-505. doi: 10.1007/978-1-4757-5348-6_31

61. Sapermsap N, Li DDU, Al-Hemedawi R, Li Y, Yu J, Birch DJ, et al. A rapid analysis platform for investigating the cellular locations of bacteria using twophoton fluorescence lifetime imaging microscopy. Methods Appl Fluoresc. (2020) 8:034001. doi: 10.1088/2050-6120/ab854e

Conflict of Interest: The authors declare that the research was conducted in the absence of any commercial or financial relationships that could be construed as a potential conflict of interest.

Copyright $\odot 2020$ Li, Natakorn, Chen, Safar, Cunningham, Tian and Li. This is an open-access article distributed under the terms of the Creative Commons Attribution License (CC BY). The use, distribution or reproduction in other forums is permitted, provided the original author(s) and the copyright owner(s) are credited and that the original publication in this journal is cited, in accordance with accepted academic practice. No use, distribution or reproduction is permitted which does not comply with these terms. 


\section{APPENDIX}

Derivation of $\tau_{C M M}$. Take the integration of $t \cdot h(t)$ and $h(t)$,

$$
\begin{aligned}
\int_{0}^{\infty} t \cdot h(t) d t & =\int_{0}^{\infty} t \int_{0}^{\infty} \operatorname{irf}\left(t-t^{\prime}\right) \cdot f\left(t^{\prime}\right) d t^{\prime} d t \\
& =\int_{0}^{\infty} \int_{0}^{\infty}\left(t-t^{\prime}\right) \cdot \operatorname{irf}\left(t-t^{\prime}\right) \cdot f\left(t^{\prime}\right) d t^{\prime} d t \\
& +\int_{0}^{\infty} \int_{0}^{\infty} \operatorname{irf}\left(t-t^{\prime}\right) \cdot t^{\prime} \cdot f\left(t^{\prime}\right) d t^{\prime} d t \\
& =\int_{0}^{\infty}[t \cdot \operatorname{irf}(t)] * f(t) d t+\int_{0}^{\infty} \operatorname{irf}(t) *[t \cdot f(t)] d t \\
& =\int_{0}^{\infty} t \cdot \operatorname{irf}(t) d t \int_{0}^{\infty} f(t) d t \\
& +\int_{0}^{\infty} \operatorname{irf}(t) d t \int_{0}^{\infty} t \cdot f(t) d t \\
& \int_{0}^{\infty} h(t) d t=\int_{0}^{\infty} \operatorname{irf}(t) d t \int_{0}^{\infty} f(t) d t
\end{aligned}
$$

Dividing Equation (A1) by Equation (A2) gives

$$
\frac{\int_{0}^{\infty} t \cdot h(t) d t}{\int_{0}^{\infty} h(t) d t}=\frac{\int_{0}^{\infty} t \cdot \operatorname{irf}(t) d t}{\int_{0}^{\infty} \operatorname{irf}(t) d t}+\frac{\int_{0}^{\infty} t \cdot f(t) d t}{\int_{0}^{\infty} f(t) d t} .
$$

Then,

$$
\begin{aligned}
\tau_{C M M} & =\frac{\int_{0}^{\infty} t \cdot f(t) d t}{\int_{0}^{\infty} f(t) d t}=\frac{\sum_{i=1}^{p} q_{i} \tau_{i}^{2}}{\sum_{i=1}^{p} q_{i} \tau_{i}} \\
& =\frac{\int_{0}^{\infty} t \cdot h(t) d t}{\int_{0}^{\infty} h(t) d t}-\frac{\int_{0}^{\infty} t \cdot i r f(t) d t}{\int_{0}^{\infty} i r f(t) d t} \\
& \simeq \frac{\sum_{m=0}^{M-1} t_{m} \cdot h_{m}}{\sum_{m=0}^{M-1} h_{m}}-\frac{\sum_{m=0}^{M-1} t_{m} \cdot i r f_{m}}{\sum_{m=0}^{M-1} i r f_{m}}
\end{aligned}
$$

